\title{
Modeling of PMU-Based Automatic Re-synchronization Controls for DER Generators in Power Distribution Networks using Modelica and the OpenIPSL
}

\author{
Biswarup Mukherjee $^{1} \quad$ Luigi Vanfretti ${ }^{2}$ \\ ${ }^{1}$ Indian Institute of Technology Bombay, India, bismuk. ece@gmail.com \\ ${ }^{2}$ Electrical, Computer, and Systems Engineering, Rensselaer Polytechnic Institute (RPI), USA, vanfrlerpi .edu
}

\begin{abstract}
Re-synchronization is traditionally coordinated between the electric power transmission network operators and power plants in an isolated portion of the grid. As the number of DER continues to increase with the rise of renewable energy sources located at the lower voltage networks, automatic re-synchronization methods that can be applied to a great number of DER are desirable. This paper describes the architecture and modeling of an automatic re-synchronization controller, which can be applied to synchronize an islanded portion of the grid by using remote measurements to drive a Distributed Energy Resource (DER) within the islanded network. The controller's re-synchronization function uses bus frequency measurements, which are derived using bus voltage phasors and a new bus frequency computation technique that can be used during the execution of dynamic simulations. This paper also introduces a new bus-angle difference control function within the re-synchronization control system, which allows monitoring the phase angle difference between two buses so to avoid unwanted re-synchronization. The effect of the angle difference control function is evaluated using a controlled circuit breaker considering different power dispatch levels of the generator in the distribution network model. Both deterministic and stochastic load models are used to analyze the performance of the automatic re-synchronization control system.
\end{abstract}

Keywords: Automatic re-synchronization controller; phase angle difference controller; power distribution network; synchrophasors; Modelica; OpenIPSL

\section{Introduction}

\subsection{Motivation}

Re-synchronization needs to be coordinated between the transmission operators and power plants in an isolated portion of the grid in order to maintain the balance between the power supply and demand. This task can be challenging when one portion of the distribution grid contains small generators having low inertia which is the case of Distributed Energy Resources (DER), such as small hydro, wind and solar power plants. As the number of DER continues to increase with the rise of renewable energy sources located at the lower voltage networks, automatic re-synchronization method that can be applied to a great number of DER are desirable. One of the main challenges with conventional synchronization techniques is to maintain a stable system operation when a disturbance occurs, during the re-synchronization process (Belyaev et al., 2015).

In addition, as reported in (Assis and Taranto, 2013), that perfect re-synchronization can only be achieved when there is no power flow through the coupling circuit breaker at the time of operation. Improper re-synchronization leads to poor power quality and reliability, diminishing energy economics (Mazloomzadeh et al., 2012).

To address these challenges, this paper presents the modeling of an automatic re-synchronization control system in a centralized control architecture that allows to reconnect two isolated power networks by exploiting synchrophasors and frequency estimates from Phasor Measurement Units (PMUs). PMUs are placed in both transmission and distribution networks to assist the automatic re-synchronization controller. All modeling has been carried out using Modelica language (Fritzson, 2004) and the OpenIPSL (Vanfretti et al., 2016) library.

\subsection{Literature Review}

Several aspects for grid synchronization have been discussed in (Blaabjerg et al., 2006) (Timorabadi and Dawson, 2006). It is described in (Belyaev et al., 2015) that improper re-synchronization may lead to damaging circuit breaker contacts, mechanical stresses on a generator and prime mover, and other unwanted wear/tear.

The previously cited searches also highlight that, there will be a sudden active power flow at the time of resynchronization, until the initial frequency difference of the two sides of the circuit breaker reach a stable common frequency. The other criterion is the magnitude of the voltage differences across the circuit breaker. If this magnitude is very high, it will cause sudden transient current to flow through the circuit breaker, resulting in high reactive power flow. Meanwhile it is reported in (Belyaev et al., 2015) that phase angle difference is another important criterion for re-synchronization. Therefore control of the phase angle difference across the circuit breaker can 
be beneficial for re-synchronization.

To automate the re-synchronization process, it is proposed in (Assis and Taranto, 2013) to use a voltage and speed control strategy for automatic re-connection using remote sensing of voltage and frequency signals after an intentional islanding occurs. However, this approach still depends on conventional synchronism check relays. Autosynchronizers are also available to perform automatic resynchronization when the synchronization function is active. They will automatically adjust the speed of the generator through the governor in order to match the bus frequency. This arrangement is ideal for synchronizing an islanded system of multiple generators and load to the grid or another islanded network (Thompson, 2012). Alternatively, the use of frequency estimates from PMUs are proposed in (Kirkham et al., 2014). Reference (Almas and Vanfretti, 2016) proposes to use PMUs to obtain frequency measurements and GOOSE messaging for control, however, the control actions are discretized and applied using a look-up table, which can introduce undesirable perturbation to the generator.

Alternatively, to perform re-synchronization operation, it would be equally interesting to develop a controller capable of using the frequency estimates from PMUs while applying smoother control actions. Therefore this paper proposes to use PMU measurements from both transmission or distribution networks for the re-synchronization control system, while applying smooth control actions on error signals from voltage, frequency and angle differences.

\subsection{Paper Contributions}

The main contributions of this paper are as follows:

- A bus-angle difference-based controller that helps reducing the bus voltage angle difference and enhances operation of the re-synchronization process. The controller uses the unwrapped angle calculated from the bus angles, which is required due to the angle switching.

- The proposed control system is interfaced in cascade with traditional turbine governor and excitation controllers (i.e. governor and voltage regulators) supplementing generator control systems model instead of replacing existing ones.

- The performance of the proposed control system is evaluated for both stochastic and deterministic load models to illustrate the impact of load uncertainties in the short time-scale of the re-synchronization process.

The remainder of this paper is organized as follows. In Sections 2 the centralized control system architecture and modeling of the automatic re-synchronization controller is explained. Section 3 describes the power system and simulation execution models. Finally, case studies are analyzed in Section 4 and conclusions are drawn in Section 5 .

\section{The Re-synchronization Controller}

\subsection{Control Architecture}

A schematic of the proposed control architecture for automatic re-synchronization is shown in Figure 1. It contains three major functions or units: computation unit, activation unit and control unit. The computation unit computes the bus voltage, frequency and angle differences. The control system is comprised of a voltage controller, a frequency controller and an angle-difference controller. In addition the 'activation unit' is used to trigger the individual regulators following different control modes.

The decisions taken by this unit require checking thresholds for three different synchronization variables: $\Delta \mathrm{V}, \Delta \mathrm{F}, \Delta \theta$; these are the voltage, frequency and angle differences, respectively, obtained from the computation unit. The synchronizing variables are calculated using PMU measurements from two locations of the power network; one is the main grid and one in the islanded grid under control.

One of the control modes of the activation unit will be analyzed herein the traditional sequential mode. This mode activates each of the individual controllers in sequence, after each of the individual thresholds for the synchronization variables have been reached. The order is $\Delta \mathrm{V}, \Delta \mathrm{F}$ and finally $\Delta \theta$. Once the thresholds set for the synchronization variables have been reached, the resynchronization process is completed by sending a trip signal to the receiving end of the controlled circuit breaker. Throughout this paper it is assumed that the sending end circuit breaker is closed and that the power line is energized. Switching transients (i.e. those involved in the electro-magnetic behaviour of the system) are ignored, as it is assumed that because the control system minimizes errors in the synchronizing variables, the switching transients will be negligible.

\subsection{The $\Delta V, \Delta \mathbf{F}$ and $\Delta \theta$ controller}

Similarly to (Assis and Taranto, 2013), both the voltage controller and the frequency controller use a PI block. In addition an angle difference controller, proposed here, also requires a PID function. The schematic of voltage controller and the frequency controller are shown in Figures 2 and 4 respectively while their Modelica implementations are shown in Figures 3 and 5 respectively.

Table 1. Truth table of the voltage controller

\begin{tabular}{|c|c|}
\hline Boolean Input signal & Output (y) \\
\hline True & $\mathrm{y}=\Delta \mathrm{V}\left(\mathrm{K}_{P}+\frac{K_{I}}{S}\right)$ \\
\hline False & 0 \\
\hline
\end{tabular}

In the Modelica implementation of the voltage controller (see Figure 3) u1 and u2 represent the voltage magnitudes of the transmission and distribution network buses, respectively. The output of the voltage controller is 


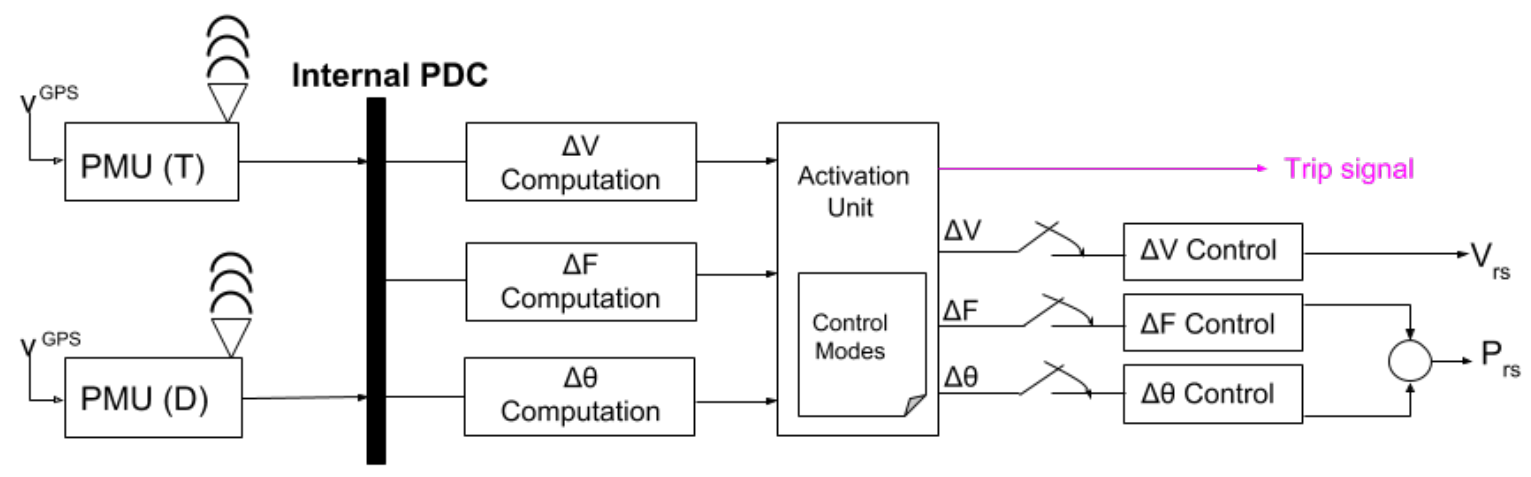

Figure 1. Architecture of the automatic re-synchronization controller.

Transmission network bus voltage

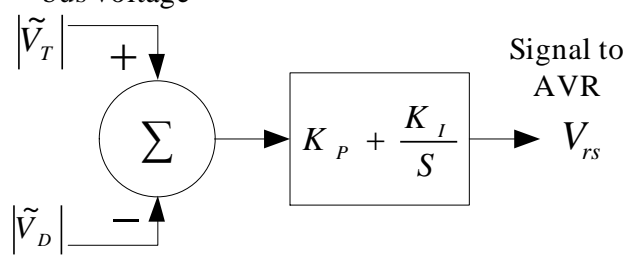

Distribution network bus voltage

Figure 2. Schematic of voltage controller.

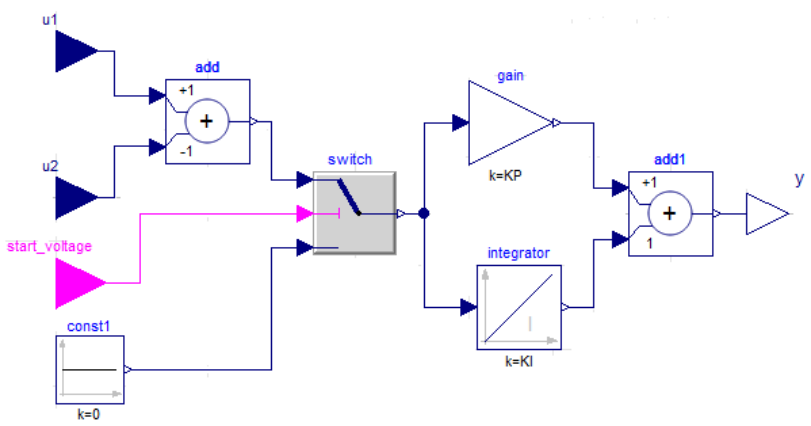

Figure 3. Implementation of the voltage controller in Modelica.

applied to the AVR error signal, which controls the field voltage of the generator.

If the Boolean input signal, start_voltage, is applied to the switch is true then the computed voltage difference is fed to the PI block. If this Boolean signal is false, the output of the controller becomes zero. In sum, the voltage controller output is defined by the truth table shown in Table 1.

In the Modelica implementation of the frequency controller (see Figure 5) f_IB and f_DN represent the frequencies of the transmission and distribution networks, re-
Transmission network bus frequency

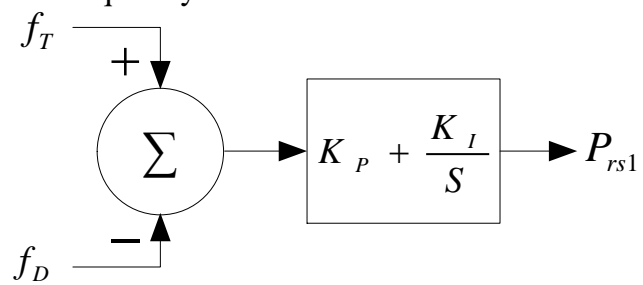

Distribution network

bus frequency

Figure 4. Schematic of frequency controller.

Table 2. Truth table of the frequency controller

\begin{tabular}{|c|c|}
\hline Boolean Input signal & Output (y) \\
\hline True & $\mathrm{y}=\Delta \mathrm{f}\left(\mathrm{K}_{P}+\frac{K_{I}}{S}\right)$ \\
\hline False & 0 \\
\hline
\end{tabular}

spectively. The output of the frequency controller is Prs 1 as shown in Figure 5.

If the Boolean output signal from the XOR gate is true, the output of the switch is the frequency difference, which is applied to the input of the PI controller. If this Boolean output of the XOR gate is false then the output of the switch is zero, and as a result, the output of the PI controller becomes zero. The output of the XOR gate becomes true when either of the Boolean inputs is true, if booth the inputs are true then output is false. The Boolean signal Trigger turns true when the voltage limit is satisfied. The Boolean signal Block is true when all three $(\Delta \mathrm{V}, \Delta \mathrm{F}$ and $\Delta \theta)$ limits are checked successfully. Therefore the output of the frequency controller can be represented as shown in Table 2.

The angle difference controller is a PID controller whose input is the angle difference between the transmission and distribution side bus voltage angles. A simple 


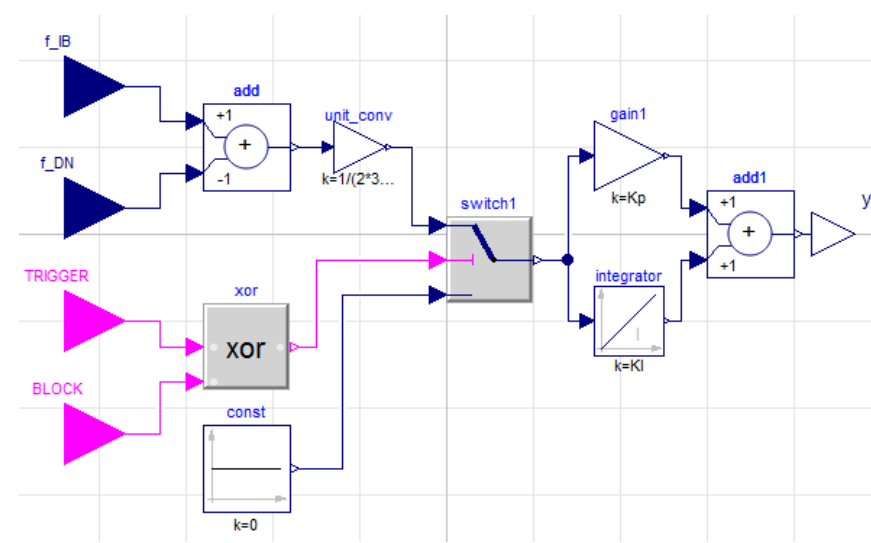

Figure 5. Implementation of the frequency controller in Modelica.

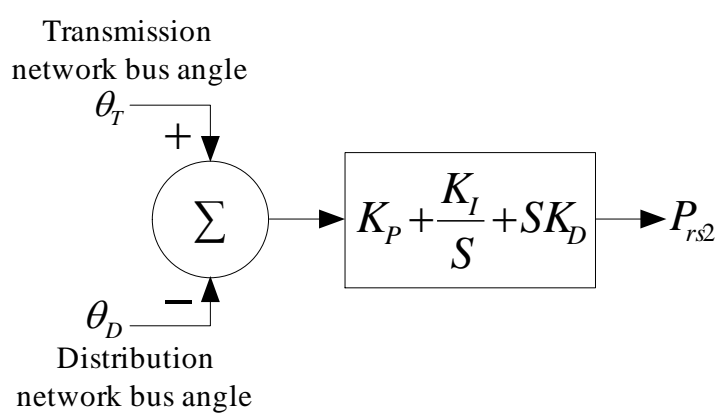

Figure 6. Schematic of $\Delta \theta$ controller.

block diagram representation of this controller is shown in Figure 6, while its Modelica implementation is presented in Figure 7. The output of the angle difference controller is described in Table 3.

Table 3. Truth table of the $\Delta \theta$ controller

\begin{tabular}{|c|c|}
\hline Boolean Input signal & Output (y) \\
\hline True & $\mathrm{y}=\Delta \theta\left(\mathrm{K}_{P}+\frac{K_{I}}{S}+K_{D} S\right)$ \\
\hline False & 0 \\
\hline
\end{tabular}

PMUs perform angle wrapping when reporting phasor data, if used in such representation during simulation, computations will face numerical issues (Milano and Ortega, 2017). Hence to avoid this difficulty the following Modelica code was implemented to unwrap the wrapped bus voltage angle at each bus, from where the angle difference is calculated. The angle controller uses the new angle input from theta_diff_new, so that the angle difference does not corrupt the performance of the proposed controller. The calculation of theta_diff_new uses the Modelica operator Homotopy ${ }^{1}$ that operates on the actual bus angle difference due to its non-linearity.

${ }^{1}$ Online at: http://modelica.readthedocs.io/en/latest/operators.html

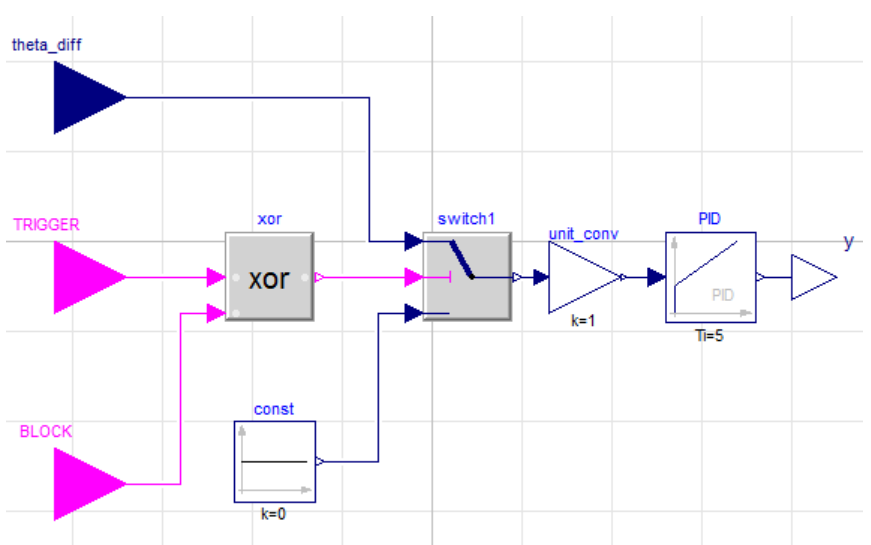

Figure 7. Implementation of the angle difference controller in Modelica.

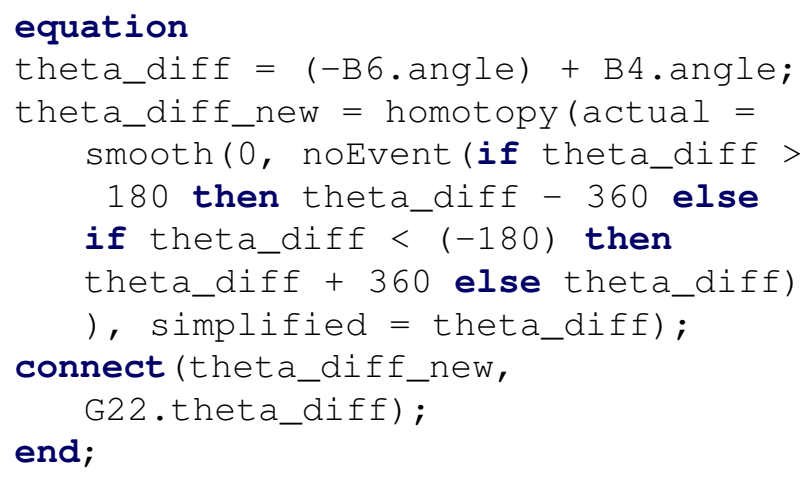

\subsection{Centralized control system architecture and Modelica implementation}

The Modelica implementation of Figure 1 is presented in Figure 8. The controller is modeled using a centralized control architecture deployed in the generator at the distribution network. The TriggeredSampler blocks are used inside the re-synchronization unit. These blocks are used from the Modelica Standard Library ${ }^{2}$ and latch the input when the Boolean trigger input signal is true. The Boolean output signal from the re-synchronization unit is applied to the circuit breaker when all three limits are satisfied inside the activation unit. The Modelica implementation of the generator at the distribution network is presented in the Figure 11.

\subsection{Modeling of Frequency computation Block}

In previous work (Mukherjee and Vanfretti, 2018), the authors developed a technique to compute frequency estimates during the execution of dynamic simulation. Simulation results comparing this model with the conventional frequency computation approach used in power systems. This previous results are used in this work.

To briefly summarize this previous work, let $\omega$ be frequency of the bus voltage, the first order derivative of the bus angle represents the frequency deviation at the bus.

${ }^{2}$ Online at: https://github.com/modelica/ModelicaStandardLibrary 


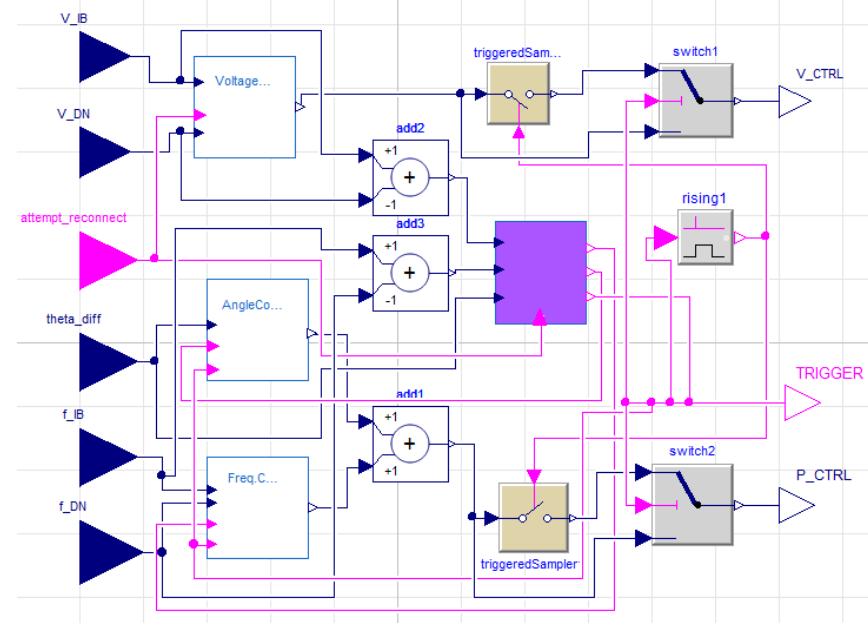

Figure 8. Implementation of the automatic re-synchronization controller in Modelica.

The bus frequency can be determined from the following equation, where $V_{r}$ and $V_{i}$ represent the real and imaginary parts of complex bus voltage respectively:

$$
\omega=\frac{V_{r} \dot{V}_{i}+V_{i} \dot{V}_{r}}{V_{i}^{2}+V_{r}^{2}}
$$

The controller uses the equation above to compute the bus frequency from the bus voltage data, which is implemented as a Modelica code in the frequency computation blocks inside the simulation set-up that will be described in the next sections.

\section{Power System Model and Simula- tion Set-up Implementation}

\subsection{Power System Model Implementation}

Figure 9 shows the power system model mapped on the component layer of Smart Grid Architecture Model (Hooshyar and Vanfretti, 2017), while it's Modelica implementation presented in Figure 10. Figure 9 is useful to understand re-synchronization would require the coordination between three domains - transmission, distribution, and DER owners, and thus, the proposed resynchronization controller would be beneficial. The figure shows how PMU data is measured at both a transmission and distribution substation, while the re-synchronization controller is located at the DER substation.

In the Modelica model shown in Figure 10, the resynchronization controller is within the generator (G22). This is expanded in Figure 11, that shows how G22 is modeled using the GENSAL block in OpenIPSL that corresponds to the synchronous generator; IEEESGO is the OpenIPSL block that corresponds to the gas and turbine model, and SEXS is the OpenIPSL block used to model the excitation control system of the generator. Meanwhile, in Figure 10, G1 represents a large power plant connecting to the transmission portion. This is expanded in Fig- ure 12, that shows how G1 is modeled using the HYGOV block from OpenIPSL that corresponds to a hydraulic turbine and governor systems model (HYGOV). To model hydraulic power plants, the synchronous generator GENSAL block in OpenIPSL is used to consider salient fluxes, and excitation control system of the generator (SEXS). The upper left corner of Figure 12 shows how the overall system frequency is varied by introducing a speed change in the governor system of the transmission network generator model, before the re-synchronization process starts.

\subsection{Simulation Set-Up Implementation}

The Modelica implementation of the simulation set-up block is presented in Figure 13. It is used to set-up the re-synchronization event that leads to the activation of the automatic re-synchronization controller. The circuit breaker 2 remains always closed and keeps line (L3) energized from the transmission side. Circuit breaker 1 is controlled with the following logic. Initially, for first 6 seconds, the breaker is closed and after that it is open. At 6.01 seconds the re-synchronization process starts and the Boolean signal y 3 becomes true. This output is applied as the Boolean input to the automatic re-synchronization unit to start the voltage control. When the Boolean output from the activating unit becomes true, the breaker CB2 is closed. The frequency computation blocks calculate the frequencies of each side of the network. The outputs $y 1$ and y 2 from these frequency calculation blocks are applied to controller inside G22, while a Boolean constant true signal keeps the circuit breaker CB1 closed to keep the transmission line energized between both breakers.

\section{Case Studies}

The following case studies are performed using a steam turbine and governor system in the distribution side generator model G22 to analyze the performance of the proposed automatic re-synchronization controller.

\subsection{Sequential Control Mode Performance}

This case study includes the performance of the resynchronization controller shown in Figures 14-16. As it can be observed from Figure 14a, soon as the resynchronization process starts at 6.01 seconds the voltage controller starts working and effectively reduces the oscillations in bus voltages (Bus 4 and Bus 6 voltage). After the re-synchronization occurs at 150 seconds the voltage controller still works minimizing the error in the voltage difference for both the buses (Bus 4 and Bus 6).

From Figure 15a it can be seen that both the transmission and distribution network frequency deviation undergo excursion of $-0.5 \leq \Delta \mathrm{f}_{T} \leq-0.5$ and $-0.4 \leq \Delta \mathrm{f}_{D} \leq-0.05$. The frequency controller in this paper aims to reduce the frequency deviation to zero and that can be seen from Figure $14 \mathrm{~b}$; after the network is re-connected with the transmission side, frequency deviation (with respect to that of the transmission network) remains zero which satisfies the goals of the proposed automatic re-synchronization con- 


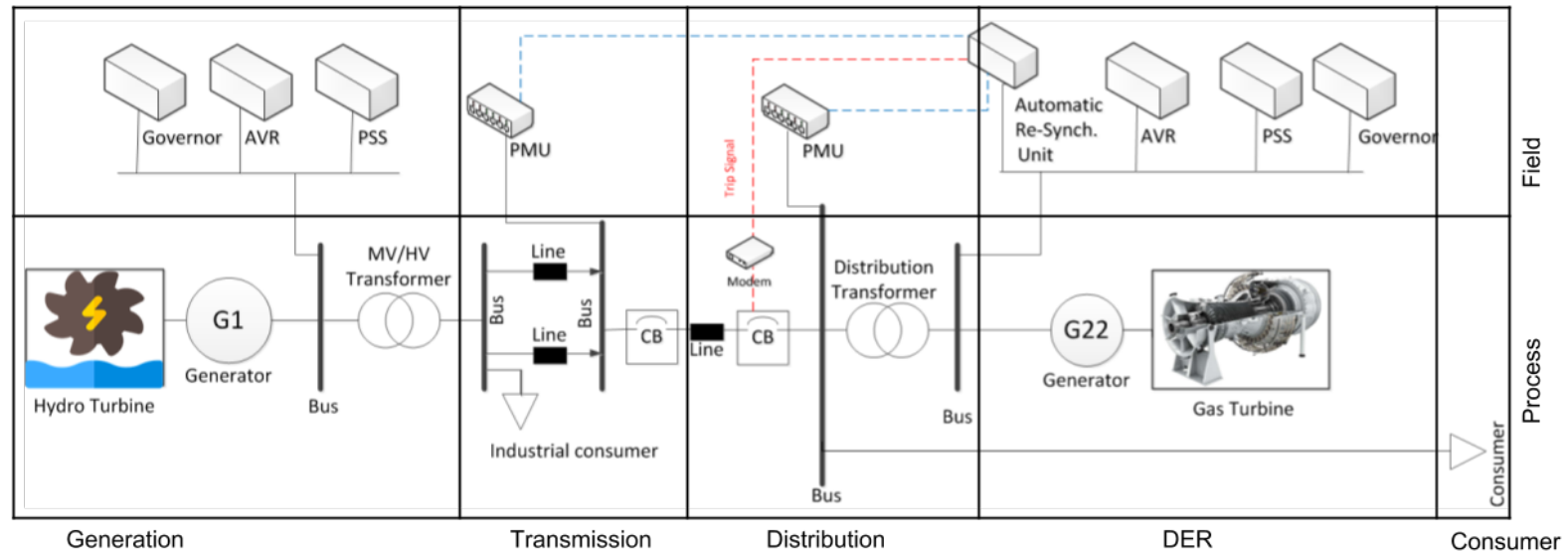

Figure 9. The use case mapped on the SGAM component layer.

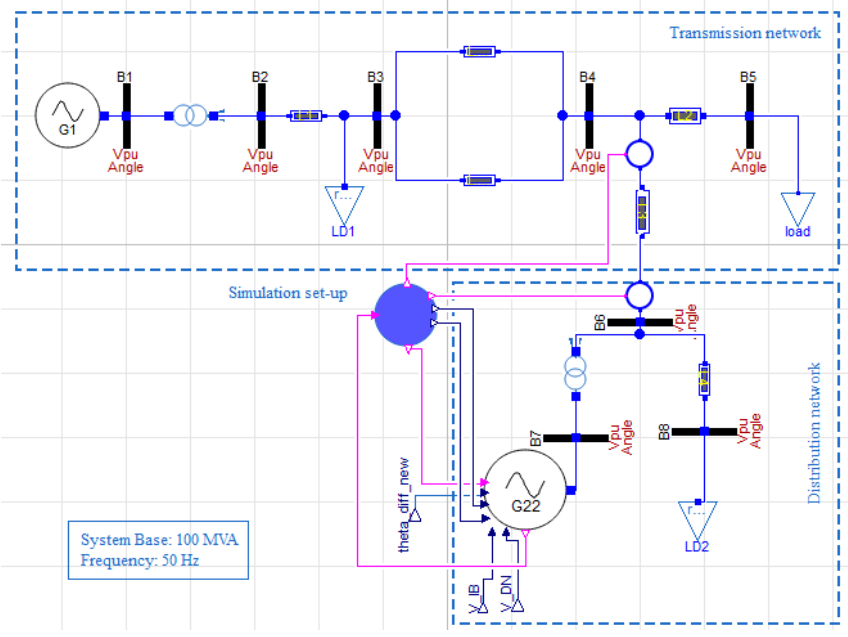

Figure 10. Power network models using components from OpenIPSL in Modelica.

troller. In Figure 16a the phase angle difference of the bus voltages are plotted where it can be seen that during and after re-synchronization the re-synchronization the bus voltage angle difference also remains zero, implying ideal automatic re-synchronization. Figure $16 \mathrm{~b}$ plots the triggered signal to the controlled circuit breaker CB2.

\subsection{Performance of the Angle Control Func- tion}

When measured by PMUs the angle of the bus voltage phasor switches between $+/-\pi$ (+/- 180 degrees), therefore the angle difference calculated directly from bus data the may corrupt the performance of the angle controller if it is wrapped. This case study exhibits the performance of the angle control for both wrapped and unwrapped angle calculations for a dispatch of $10 \mathrm{MW}$ from generator G22, as shown in Figure 17. The the angle controller is activated at $\mathrm{t}=101$ seconds whereas re-synchronization process starts at $\mathrm{t}=6.01$ seconds . As it can be observed from the red trace, the unwrapped angle difference calculated from the

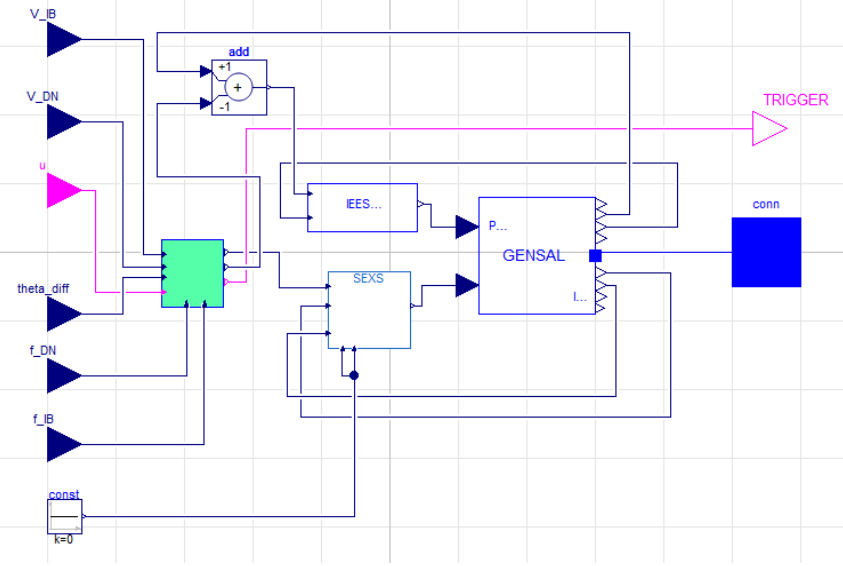

Figure 11. Centralized control structure within the generator model (G22) implemented using Modelica.

wrapped angle differences produces no transients in its response, which makes the angle controller effective during the automatic re-synchronization process.

\subsection{Effect of Angle Difference Controller dur- ing the Re-synchronization Process}

This case study is performed to illustrate the effect of angle difference on the total time taken for resynchronization, and also to understand the effect of angle controller on the circuit breaker current magnitude at the time of automatic breaker closure.

After the voltage and frequency control have minimized their control errors below the pre-defined thresholds the output of the angle difference controller plays an important role controlling the time for the automatic breaker re-closing process. The phase angle difference and the control signal to the circuit breaker 1 (CB1), are plotted in Figure 18 for different dispatches from the distribution side generator to demonstrate this effect. As it can be observed from in Figure 18, when the angle difference between the distribution side and transmission side 


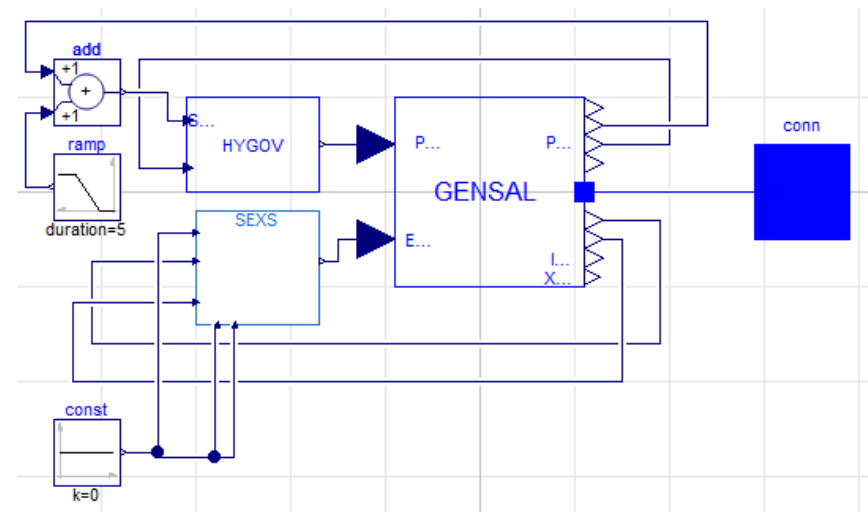

Figure 12. Implementation of the transmission network generator model (G1) in Modelica.

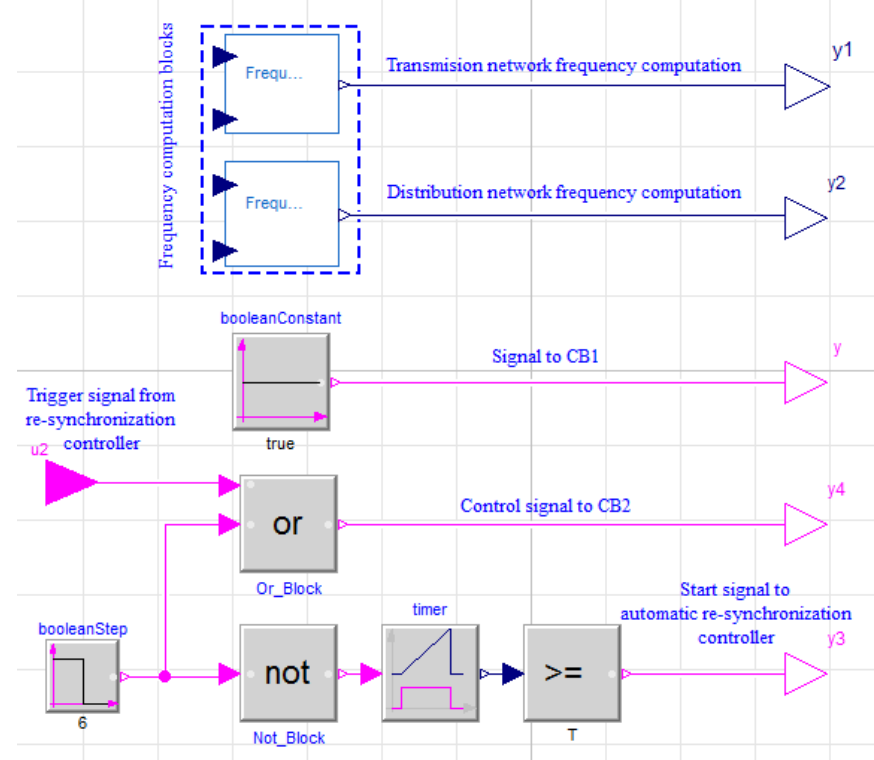

Figure 13. Modelica implementation of the simulation set-up.

bus voltage phasor reaches a steady state value, CB1 receives a trigger signal for automatic re-closure. For lower dispatches the angle difference controller is faster because the generator has a larger bandwidth (i.e. available capacity) to minimize the angle difference by speeding up the active power output. Hence there is a large value of distribution generation to (if possible) have available capacity resources to use for frequency ancillary services during resynchronization to the transmission grids.

From Figure 19, it can be observed that with angle difference control activated the magnitude of the circuit breaker CB2 current reduces, which implies that presence of the angle control unit reduces unwanted effect of improper re-synchronization. This comes at the cost of a longer resynchronization time, however, note that this is the time spent on minimizing $\Delta \theta$.

\subsection{Effect of Stochastic Load Model}

In this case the performance of the re-synchronization controller is analyzed for both deterministic and stochastic

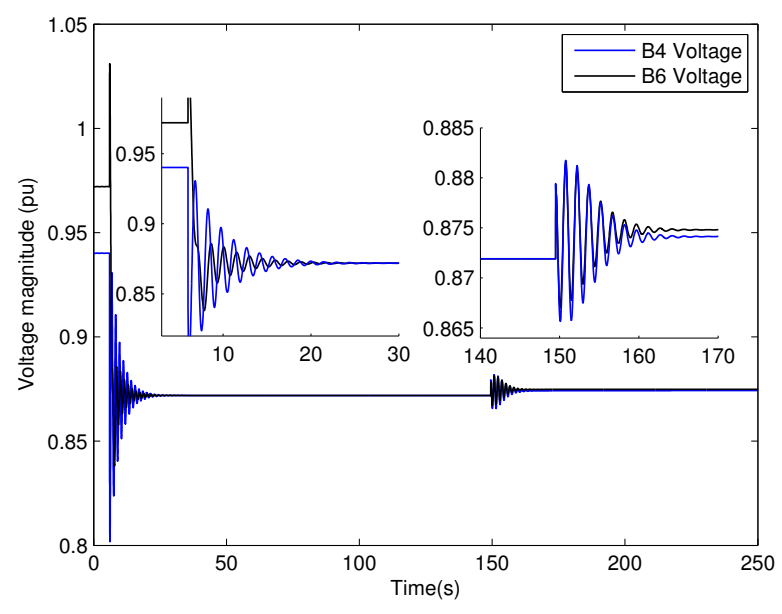

(a) Transmission and distribution network bus voltages.

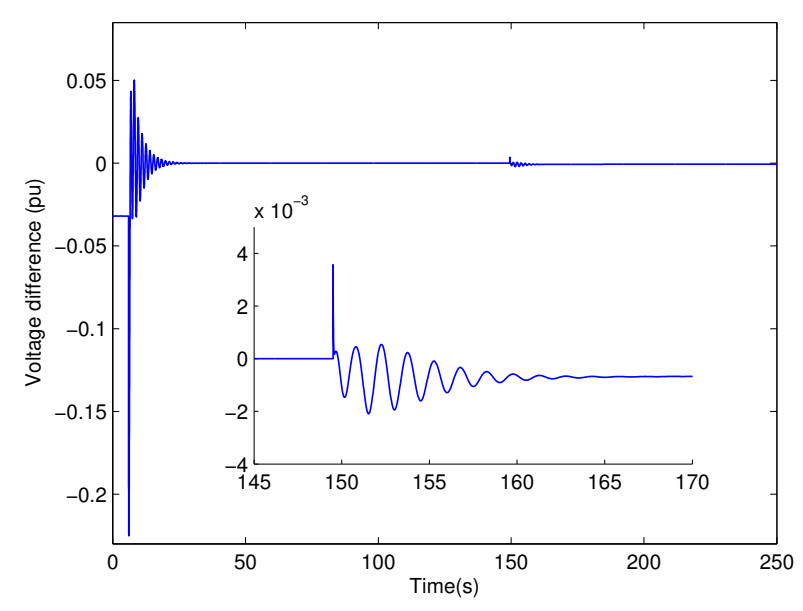

(b) Voltage difference during re-synchronization.

Figure 14. Bus voltages and bus voltage difference during the re-synchronization process for 10MW dispatch from G22.

load models. Figure 20 shows the plot of the transmission side frequency for both models. As it can be observed, when the stochastic load model introduces uncertainties in the load response, which in turn, affect the voltage phasor values. As a result, the estimated frequency will vary, and consequently, the controller activation time can no longer be determined or designed deterministically. Depending on the variance used for the stochastic load model different answers can be obtained. What this aims to show is the need of stochastic modeling when considering renewable energy sources (RES), as the different thresholds need to be determined a-priori, they need to be computed probabilistically using the stochastic model and a MonteCarlo like approach; note that this is NOT in today's current practice. This will be subject to future work.

\section{Conclusions}

The following conclusions can be drawn from the above work. The angle difference control function is required to 


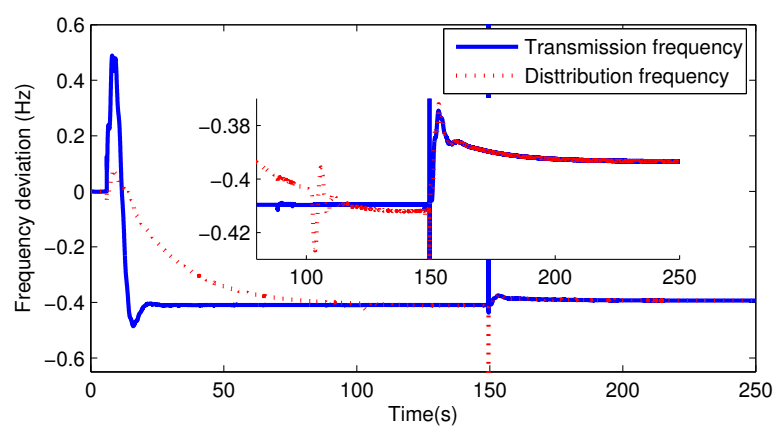

(a) Plot of frequency deviation in both transmission and distribution network

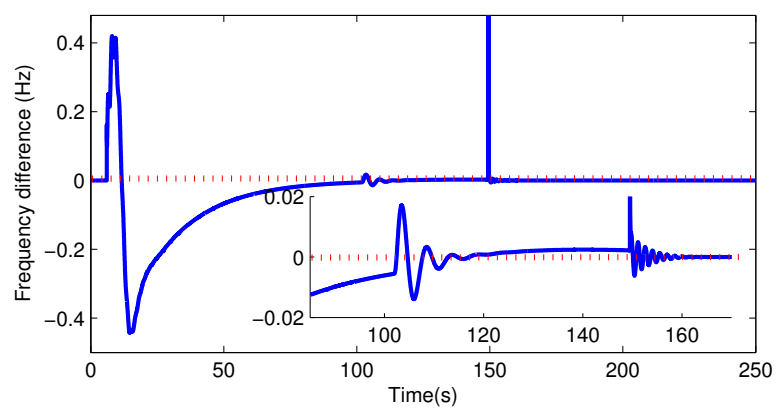

(b) Plot of frequency difference

Figure 15. Frequency deviation and frequency difference during re-synchronization for 10MW dispatch from G22.

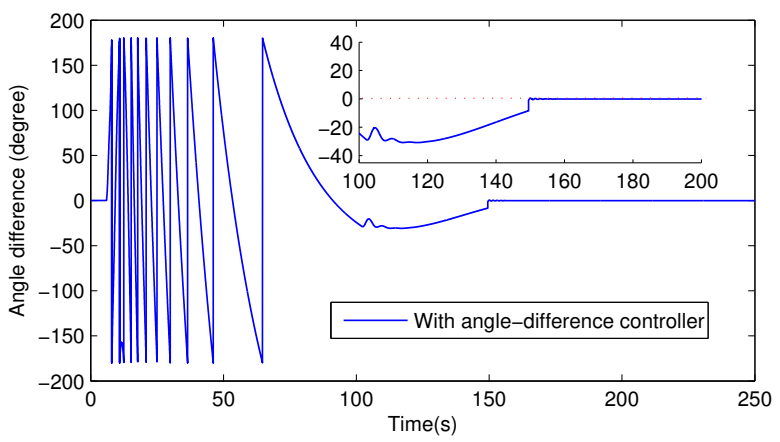

(a) angle difference

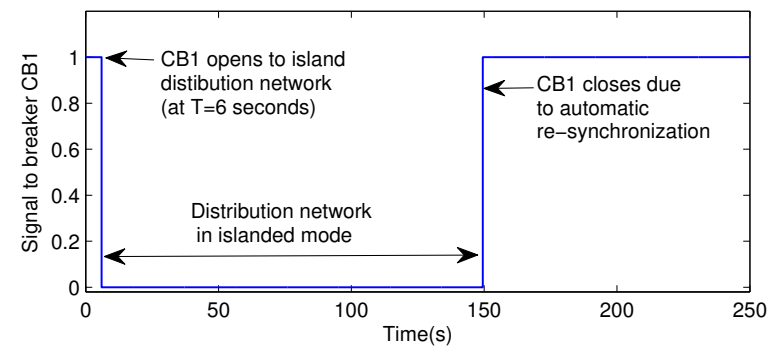

(b) signal to breaker CB1

Figure 16. Plot of angle difference and controlled signal to CB1 during re-synchronization for 10MW dispatch from G22

perform seamless automatic re-synchronization process, hence, reducing the circuit breaker power at the time of re-connection. It is necessary to unwrap the bus voltage

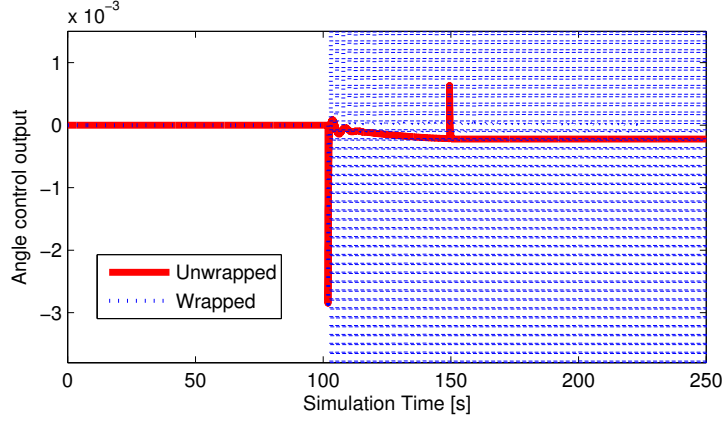

Figure 17. Performance of the angle difference controller due to angle measurement unwrapping.

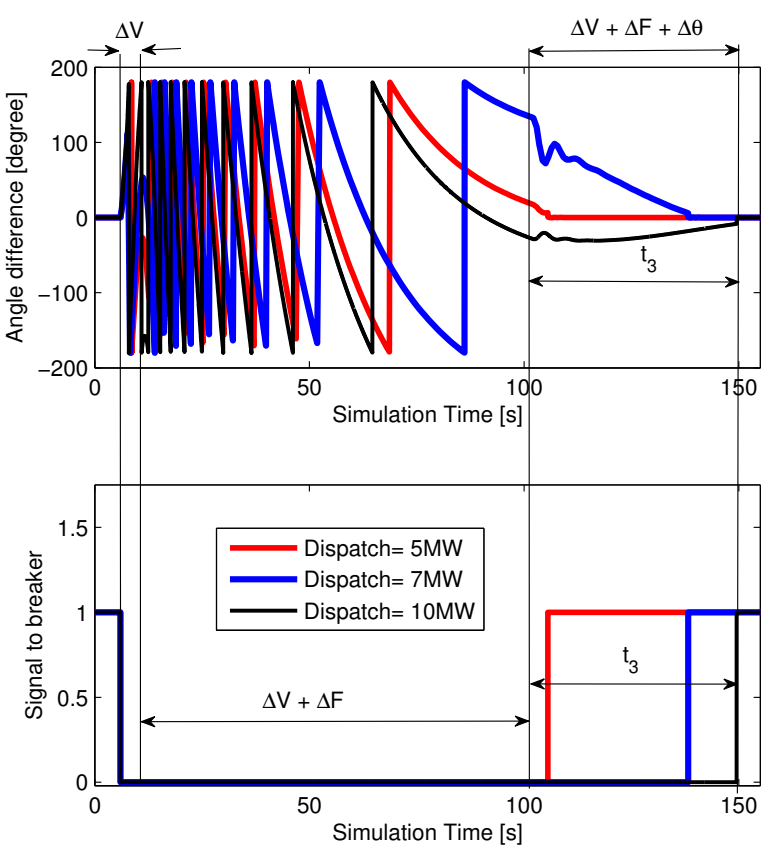

Figure 18. Angle difference controller effect on the resynchronization time for different dispatches.

phase angle otherwise the wrapped bus angle will corrupt the performance of the angle controller. The architecture of the automatic re-synchronization controller performs satisfactorily for both deterministic and stochastic load models.

Further work should involve the performance analysis of the automatic re-synchronization controller for different reporting rates, and data transmission delays for PMU devices. It will be also be interesting to investigate the performance of this controller for different of load uncertainties (noise level) in order to analyze the impact of uncertainties on the system behaviour. Authors of this paper are currently working towards these goals.

\section{Reproducible Research}

The models used to obtain the results in this paper are available online on the following Github repository: https://github.com/ 

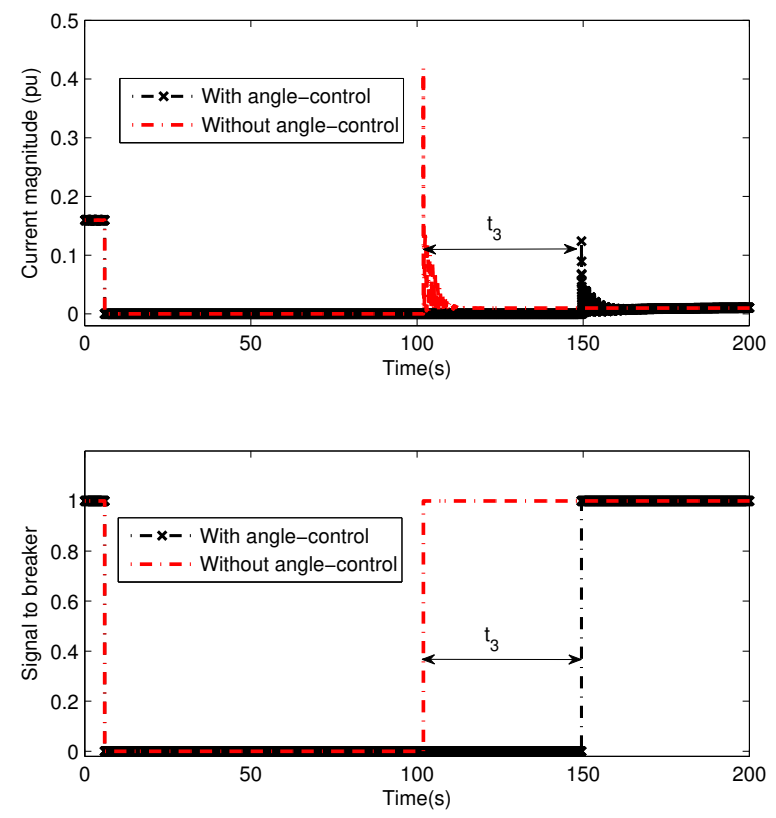

Figure 19. Circuit breaker current magnitude and trigger signal to breaker with and without angle control for 10MW dispatch.

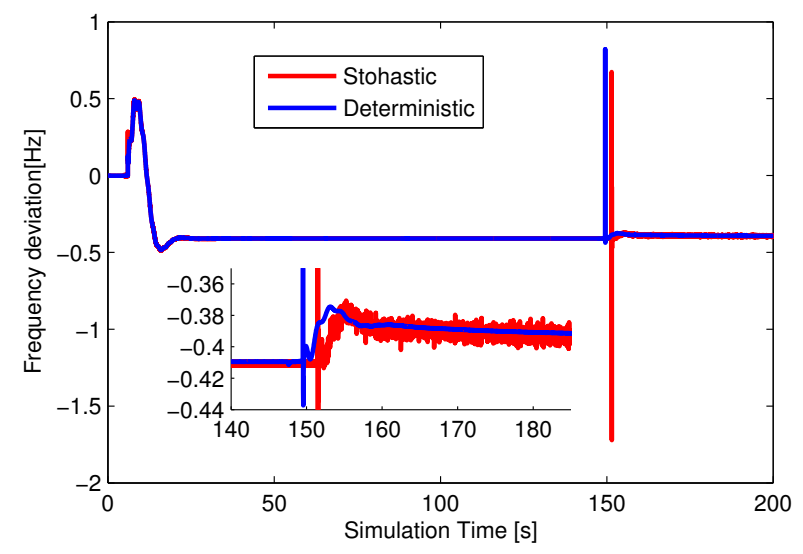

Figure 20. Transmission network frequency deviation with both deterministic and stochastic load models.

\section{ALSETLab/2019_13thModelicaConf_ PMUBasedAutomaticRe-synchronization}

\section{Acknowledgment}

Biswarup Mukherjee was supported by MHRD, Government of India, for FOSSEE-Phase II at IIT Bombay. The work of L. Vanfretti is supported in part by the Engineering Research Center Program of the National Science Foundation and the Department of Energy under Award EEC-1041877 and in part by the CURENT Industry Partnership Program.

\section{References}

M. S. Almas and L. Vanfretti. RT-HIL implementation of the hybrid synchrophasor and GOOSE-based passive islanding schemes. IEEE Transactions on Power Delivery, 31(3):1299-1309, June 2016. ISSN 0885-8977. doi:10.1109/TPWRD.2015.2473669.

T. Assis and G. Taranto. Automatic reconnection from intentional islanding based on remote sensing of voltage and frequency signals. In 2013 IEEE Power Energy Society General Meeting, pages 1-1, July 2013. doi:10.1109/PESMG.2013.6672101.

N. A. Belyaev, Y. V. Khrushchev, S. V. Svechkarev, A. V. Prokhorov, and L. Wang. Generator to grid adaptive synchronization technique based on reference model. In 2015 IEEE Eindhoven PowerTech, pages 1-5, June 2015. doi:10.1109/PTC.2015.7232582.

F. Blaabjerg, R. Teodorescu, M. Liserre, and A. V. Timbus. Overview of control and grid synchronization for distributed power generation systems. IEEE Transactions on Industrial Electronics, 53(5):1398-1409, Oct 2006. ISSN 0278-0046. doi:10.1109/TIE.2006.881997.

Peter Fritzson. The Modelica Language. IEEE, 2004. doi:10.1109/9780470545669.part2. URL

https: //ieeexplore.ieee.org/xpl/

articleDetails.jsp?arnumber $=5264368$.

H. Hooshyar and L. Vanfretti. A sgam-based architecture for synchrophasor applications facilitating tso/dso interactions. In 2017 IEEE Power Energy Society Innovative Smart Grid Technologies Conference (ISGT), pages 1-5, April 2017. doi:10.1109/ISGT.2017.8085977.

Harold Kirkham, Jeffery E. Dagle, and Y. Sun. PMU measurement technology. Technical report, Pacific Northwest National Laboratory (PNNL), 04/2014 2014.

A. Mazloomzadeh, V. Salehi, and O. Mohammed. Soft synchronization of dispersed generators to micro-grids for smart grid applications. In 2012 IEEE PES Innovative Smart Grid Technologies (ISGT), pages 1-7, Jan 2012. doi:10.1109/ISGT.2012.6175812.

F. Milano and A. Ortega. Frequency divider. IEEE Transactions on Power Systems, 32(2):1493-1501, March 2017. ISSN 0885-8950. doi:10.1109/TPWRS.2016.2569563.

B. Mukherjee and L. Vanfretti. Modeling of PMU-based islanded operation controls for power distribution networks using Modelica and openIPSL. In Proceedings of The American Modelica Conference, MA, USA, October 2018.

M. J. Thompson. Fundamentals and advancements in generator synchronizing systems. In 2012 65th Annual Conference for Protective Relay Engineers, pages 203-214, April 2012. doi:10.1109/CPRE.2012.6201234.

H. S. Timorabadi and F. P. Dawson. A wide-range synchronization system for AC power systems. In 2006 IEEE International Symposium on Industrial Electronics, volume 3, pages 1667-1672, July 2006. doi:10.1109/ISIE.2006.295820.

L. Vanfretti, T. Rabuzin, M. Baudette, and M. Murad. itesla power systems library (iPSL): A Modelica library for phasor time-domain simulations. SoftwareX, 5:84 - 88, 2016. ISSN 2352-7110. doi:http://dx.doi.org/10.1016/j.softx.2016.05.001. 
Modeling of PMU-Based Automatic Re-synchronization Controls for DER Generators in Power Distribution Networks using Modelica and the OpenIPSL 